\title{
Hyperon-nucleon femtoscopy, nuclear production and bearing on astrophysics
}

\author{
Valentina Mantovani Sarti ${ }^{1, *}$ \\ ${ }^{1}$ Physik Department E62, Technische Universität München, James-Franck-Str. 1, 85748, Garching, Ger- \\ many
}

\begin{abstract}
The presence of hyperons in the inner core of neutron stars is still an open issue in astrophysics and the so-called hyperon puzzle is far away from being solved. A complete understanding of how the interaction between hyperons and nucleons, at the level of two- and three-body, behaves in vacuum and at finite baryon density is a key requirement in order to build a realistic Equation of State for matter inside the core of neutron stars. Recently, measurements of hadron-hadron correlation functions with the femtoscopy technique in small colliding systems delivered a large amount of precise data on several hyperonnucleon pairs. Several interactions relevant for the physics of neutron stars, such as $\mathrm{p} \Lambda$ and $\mathrm{p} \Xi$, have been compared to the femtoscopic data and the effects on the corresponding Equation of State have been investigated. The extension to the three-body forces with the measurement of correlations involving triplets and proton-deuteron pairs will provide for the first time access to the genuine three-body interaction and shed light on the hyperon-puzzle.
\end{abstract}

\section{Introduction}

The interaction of hyperons, hadrons with one of more strange quarks, with nucleons is one of the key ingredients needed to understand the composition of the most dense objects in our universe: neutron stars (NS) [1]. These kinds of stars are characterized by large masses $\left(M \approx 1.5-2.2 M_{\odot}\right)$ and small radii $(R \approx 10-15 \mathrm{~km})$ [2-4], leading to the possibility to achieve densities in the core up to several times the nuclear saturation density $\rho_{0}=0.17 \mathrm{fm}^{-3}$. The measurements of masses and radii have been so far fundamental in the investigation and modeling of the Equation of State (EoS), namely on how nuclear matter behaves in the inner core of NS. Recently, the experimental database has been increased by the addition of precise radii measurements from the NICER Collaboration $[1,5]$ and by the determination of the tidal deformability extracted from gravitational waves events involving NS systems [6,7]. The recent increase of experimental data on NS, in particular the improved precision in the measurements of the radius, opens a new chapter towards the understanding of the main constituents in the EoS and how these constituents interact in a dense environment as the core of NS.

This high density environment $\left(\rho \approx 4 \rho_{0}\right)$ supposed to occur in the interior of NS leads to an increase in the Fermi energy of the nucleons, translating into the appearance of new degrees of freedom such as hyperons [8]. The energetically favored production of strange hadrons

\footnotetext{
*e-mail: valentina.mantovani-sarti@tum.de
} 
induces a softening of the EoS which cannot be reconciled with the current observations of heavy NS, beyond $2 M_{\odot}$. The solution of this so-called hyperon puzzle is a precise understanding of the interaction between hyperons and nucleons, at the two-body (YN) and three-body (YNN) level. At the moment, however, experimental data on the genuine three-body interactions with hyperons are not yet present and the precision on the knowledge of $\mathrm{YN}$ interactions from scattering data and hypernuclei is mostly limited to the strangeness $|S|=1$ sector [8,9]. In order to build a realistic EoS, precise data on two- and three-body interactions with hyperons are needed. The measurements of correlations in the momentum space between hyperons and nucleons, performed in ultrarelativistic proton-proton (pp) collisions at the Large Hadron Collider (LHC) by the ALICE Collaboration, provided the most precise data for several YN interactions. In this contribution, the main experimental results on YN and YNN interactions, obtained by means of the femtoscopy technique, useful for the physics of NS and its EoS, will be presented.

\section{Femtoscopy at the Large Hadron Collider}

The main observable in femtoscopy is the correlation function $C\left(k^{*}\right)$. In Fig. 1, the theoretical definition given by the Koonin-Pratt formula [10] is shown in (a). One of the two main ingredients is the particle emitting source $S\left(r^{*}\right)$ (in orange in (c)) which depends on the relative distance $r^{*}$ among particles. The pair of interest is composed of particles emitted from the source which are close in the relative momentum $k^{*}$, evaluated in the pair rest frame (see [11] for details). As the particles fly towards the detector they will likely undergo final-state interaction. This is embedded in the second ingredient needed to evaluate the theoretical $C\left(k^{*}\right)$ : the modulus squared of the relative wavefunction $\psi\left(\mathbf{k}^{*}, \mathbf{r}^{*}\right)$ obtained from the solution of the Schrödinger equation [12] (in blue in (c)). Depending on the nature of the interaction, the correlation function has a specific shape, as seen in (b) and (d). In particular, in the absence of interaction the correlation function equals unity, a repulsive interaction leads to a depletion at low $k^{*}$, an attractive interaction leads to an enhancement and a bound state is presented differently based on the size of the emitting source.

From an experimental point of view, the $C\left(k^{*}\right)$ can be obtained by measuring the $k^{*}$ distribution of pairs within the same event $\left(N_{\text {same }}\left(k^{*}\right)\right)$ divided by pairs from different (mixed) collisions $\left(N_{\text {mixed }}\left(k^{*}\right)\right)$. The factor $\xi\left(k^{*}\right)$ includes the corrections due to experimental effects (e.g momentum resolution) [13]. In order to study the strong hadron-hadron interaction, typically acting at distances of $1 \mathrm{fm}$, the particles composing the pair must be emitted at similar relative distances.

\section{Accessing the strong interaction with femtoscopy in small colliding systems}

At LHC, the size of the emitting source can be varied experimentally by changing the colliding system. In the ALICE experiment, source sizes ranging from $\approx 1-1.8 \mathrm{fm}$ up to $\approx 1.8-2.5$ $\mathrm{fm}$ (ultra-peripheral and peripheral) for $\mathrm{pp}$ and $\mathrm{p}-\mathrm{Pb}$ collisions and $\approx 4-10 \mathrm{fm}$ (semi-central and central) for $\mathrm{Pb}-\mathrm{Pb}$ collisions can be probed. The study of the short-range interaction between hadrons can be directly accessed by performing femtoscopic measurements in small colliding systems as pp and $\mathrm{p}-\mathrm{Pb}$, as will be shown in Sec. 4.

In the left panel of Fig. 2 a typical Gaussian source distribution of similar size to the one obtained experimentally is shown and a typical nuclear potential is presented for comparison. As can be seen, the range of the strong interaction is relevant up to $\approx 2 \mathrm{fm}$. In this range a 


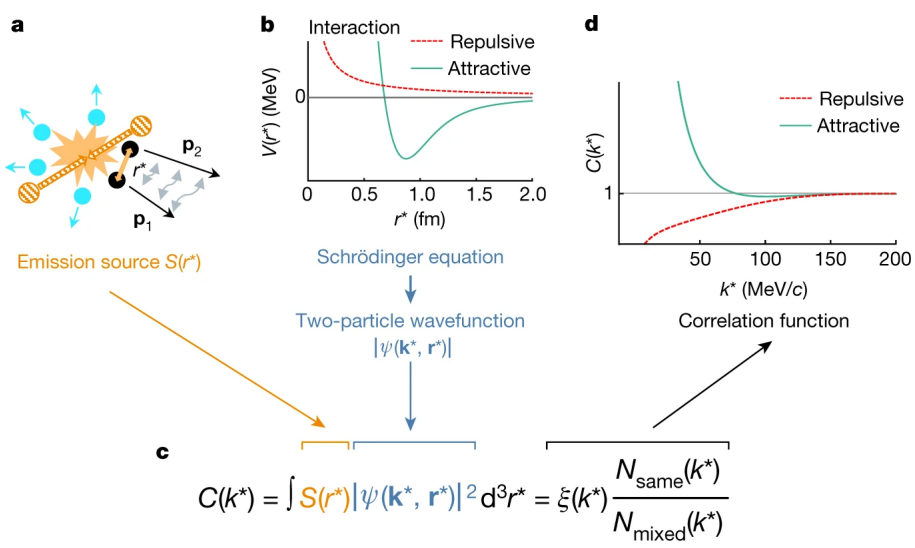

Figure 1. Figure taken from [13]. Schematic representation of the femtoscopy technique. In (a), a sketch of the source from which the pair of interest is emitted is shown, while in (b) two examples of interacting potential are presented. In (c) both the theoretical and the experimental definition of $C\left(k^{*}\right)$ are given, and finally in (d) the corresponding correlations for the two interactions in (b) are shown.

large amount of particle pairs with small $k^{*}$ can be produced, as shown by the blue shaded area.

The measurement of $C\left(k^{*}\right)$ in small colliding systems provides not only a quantitative insight into the strong interaction but it also acts as a doorway to access baryon density above $\rho_{0}$.

In order to investigate the strong interaction, a solid knowledge on the emitting source

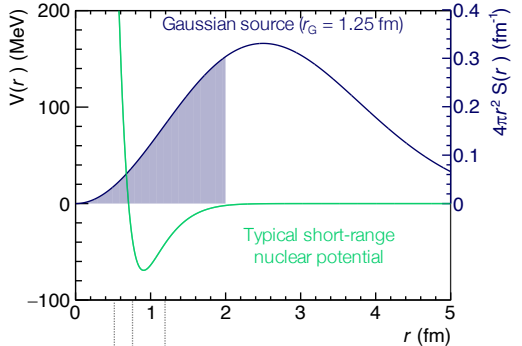

$531 \rho_{0}$

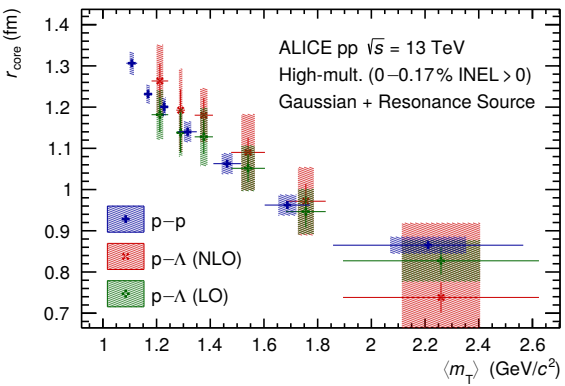

Figure 2. Left: the Gaussian source distribution (blue) for a source size of $r_{G}=1.25 \mathrm{fm}$ as a function of the relative distance, compatible with measurements in $\mathrm{pp}$ and $\mathrm{p}-\mathrm{Pb}$ collisions at LHC. In green, a typical short-range nuclear potential as a function of the relative distance. The blue shaded area represents the amount of particle pairs sensitive to the short-range interaction that can be emitted for the given source. Right: Figure taken from [14]. Source radius $r_{\text {core }}$, for $\mathrm{p}-\mathrm{p}$ (blue) and $\mathrm{p}-\Lambda$ pairs (red [15], green [16]) as a function of average $m_{\mathrm{T}}$ using a core Gaussian source with strong resonances contributions.

in small colliding systems is required. The ALICE Collaboration delivered a data-driven analysis on the modeling of the source distribution in high-multiplicity pp collisions at $\sqrt{s}=13 \mathrm{TeV}$ which quantitatively showed the existence of a common core Gaussian source 
for baryon-baryon pairs, once contributions from strongly decaying resonances are properly taken into account [14]. In the right panel of Fig. 2, the extracted core $r_{\text {core }}$ is shown as a function of the average pair's transverse mass. The analysis is performed for $\mathrm{p}-\mathrm{p}$ and $\mathrm{p}-\Lambda$ pairs since the interaction in these two systems is already well known from scattering experiments. A common scaling of the core radius is observed indicating the presence of a universal emitting source for baryons in pp collisions. These results provide a data-driven method which allows to investigate the strong interaction of any hadron-hadron pair by determining the $r_{\text {core }}$ at the corresponding $m_{\mathrm{T}}$ from the $\mathrm{p}-\mathrm{p}$ results and by including the strong resonance contributions. The total source can be approximated by an effective Gaussian [14], which for the presented measurements in Sec. 4 has an effective size in the range $r_{\text {eff }} \approx 1-1.3 \mathrm{fm}$.

\section{Results}

In this section we present the results obtained from the femtoscopic measurements of the correlation functions of YN pairs with different strangeness content.

\section{$|\mathrm{S}|=1: \mathbf{p} \Lambda$ interaction and the role of the $\mathrm{N} \Sigma$ coupling}

- The dynamics in the coupled system $\mathrm{N} \Lambda-\mathrm{N} \Sigma$ originates from the same quantum numbers and the similar mass that these two system share. The $\mathrm{N} \Lambda-\mathrm{N} \Sigma$ coupling deeply affects the behaviour of the $\Lambda$ hyperons at finite density and it has implications for the $\Lambda \mathrm{NN}$ interaction. Recent Chiral Effective field theory ( $\chi$ EFT) calculations with a weaker $\mathrm{N} \Lambda-\mathrm{N} \Sigma$ coupling strength (NLO19) [9], still reproducing scattering and hypernuclei data, predict a more attractive single-particle potential $U_{\Lambda}$ at large densities in pure neutron matter. The current experimental constraints, mostly from scattering experiments, cannot discriminate between these different chiral potentials.

The measurement of $\mathrm{p}-\Lambda$ pairs in high-multiplicity pp collisions at $\sqrt{s}=13 \mathrm{TeV}$ by the ALICE Collaboration [17] is shown in Fig. 3. These data were able to extend the kinematic range in which the interaction is tested and to achieve the highest precision currently available on this interaction $(\approx 1 \%)$. This measurement also provided the first direct observation of the opening of the $\mathrm{N} \Sigma$ channel in a two-body reaction, seen in the middle panel of Fig. 3 as a cusp-structure occurring at $k^{*} \approx 290 \mathrm{MeV} / c$. The data favours a weaker $\mathrm{N} \Lambda-\mathrm{N} \Sigma$ coupling as predicted by NLO19 calculations. Additionally, a better agreement with the measured correlation is achieved if the residual $\mathrm{p}-\Sigma^{0}$ contribution $(\approx$ $16 \%$ of the total signal) is assumed flat (right panel in Fig. 3), in agreement with the results obtained from the direct measurements of $\mathrm{p}-\Sigma^{0}$ pairs [18].

\section{$|S|=2: \mathbf{p} \Xi^{-}$interaction and test of Lattice QCD potentials}

- In the left panel of Fig. 4, the p- $\Xi^{-}$correlation function measured in high-multiplicity pp collisions at $\sqrt{s}=13 \mathrm{TeV}$ by the ALICE Collaboration is shown [13]. The data are above the Coulomb-only prediction (green line), clearly indicating the first observation of a strong attractive interaction in this system. The assumption, along with Coulomb, of a lattice potential [19], calculated at the physical quark masses, for the strong part of the interaction shows an nice agreement with the data (pink band). This scenario has also been confirmed by the identical measurement performed in $\mathrm{p}-\mathrm{Pb}$ at $\sqrt{s}=5.02 \mathrm{TeV}$ [20]. Calculations at finite density based on the same lattice potentials, validated in vacuum by the above-mentioned femtoscopic measurements, provide a slightly repulsive $U_{\Xi} \approx 6 \mathrm{MeV}$ in pure neutron matter [21], which can push the production of $\Xi$ hyperons in the core of NS to higher densities and consequently stiffen the EoS. In the left panel of Fig. 4, the obtained mass-radius relation from an EoS based on mean-field calculations [22, 23] tuned 


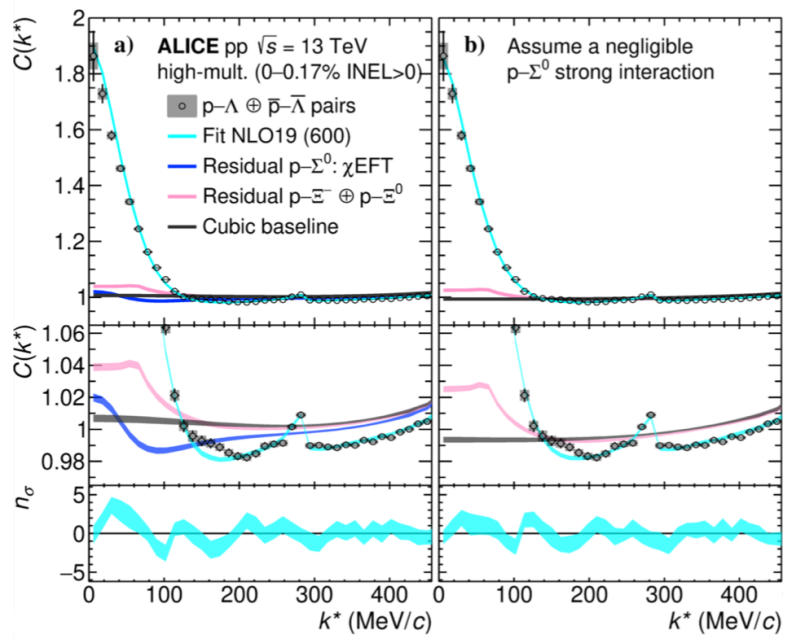

Figure 3. Figure taken from [17]. Measured $\mathrm{p}-\Lambda$ correlation and zoom in the $k^{*}$ region with the cuspstructure due to the $\mathrm{N} \Lambda-\mathrm{N} \Sigma$ coupling at $k^{*}=289 \mathrm{MeV} / c$. Lower panel: deviation between data and model in terms of $n_{\sigma}$. The fit using NLO19 [9] chiral potential is shown. Left: fit assuming a $\chi$ EFT potential [15] for the $\mathrm{p}-\Sigma^{0}$ residual. Right: fit assuming a flat $\mathrm{p}-\Sigma^{0}$ residual.

to reproduce the lattice constraint on $U_{\Xi}$ in medium is shown [11]. A maximum mass of $M=2.13 M_{\odot}$, compatible with the recent measurements [2-4] is obtained.
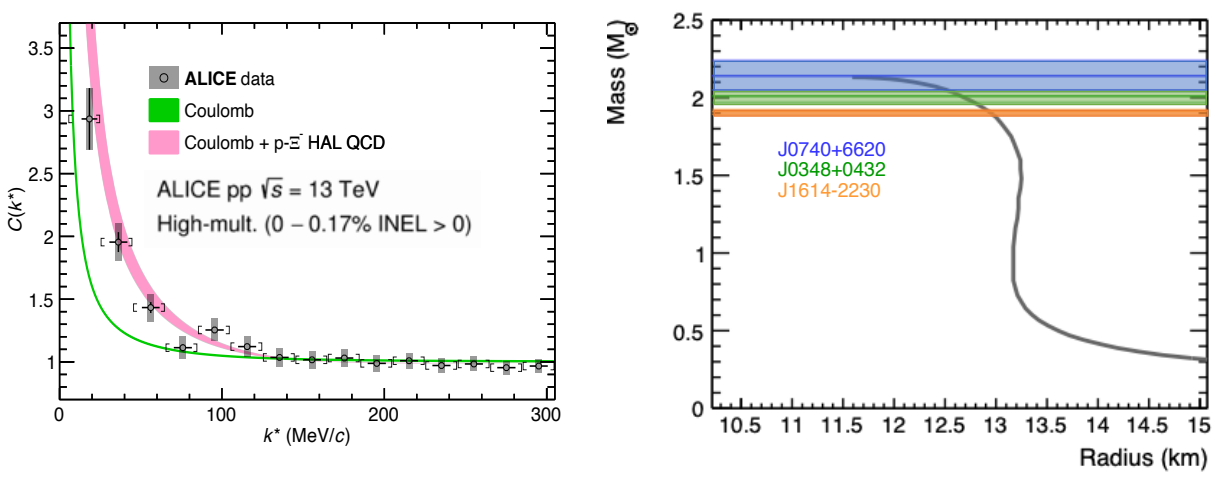

Figure 4. Left: Figure taken from [13]. Measured $\mathrm{p}-\Xi^{-}$correlation function in high multiplicity $\mathrm{pp}$ collisions at $\sqrt{s}=13 \mathrm{TeV}$ by ALICE Collaboration. The fits assuming Coulomb-only (green) and Coulomb + strong HAL QCD potentials (pink) are shown [19]. Right: mass-radius relation obtained by assuming the predicted HAL QCD $\Xi$ interaction in medium [21]. Recent astrophysical measurements of massive NS are shown in blue, green and orange [2-4].

The increased statistics expected for the upcoming Runs 3 and 4 of the LHC [24] will provide additional insights on the YN interaction and it will extend the studies to three-particle correlations in order to investigate the role played by three-body interactions, of great relevance in NS.

The access to the three-body interaction in femtoscopy will occur through a direct three- 
particle correlation measurements, by extending the two-body formalism to triplets, and through an indirect measurement, by looking at correlations between protons and composite objects such as deuterons. Recently, a projector method has been developed [25], which will allow to remove the two-body contributions from the three-body correlations. This technique will grant direct access, for the first time, to the genuine three-body interaction in $\mathrm{p}-\mathrm{p}-\mathrm{p}$ and $\mathrm{p}-\mathrm{p}-\Lambda$ systems.

\section{References}

[1] T.E. Riley et al., Astrophys. J. Lett. 887, L21 (2019), 1912.05702

[2] P. Demorest, T. Pennucci, S. Ransom, M. Roberts, J. Hessels, Nature 467, 1081 (2010), 1010.5788

[3] J. Antoniadis et al., Science 340, 6131 (2013), 1304.6875

[4] H.T. Cromartie et al., Nature Astron. 4, 72 (2019), 1904 . 06759

[5] M.C. Miller, F.K. Lamb, A.J. Dittmann, S. Bogdanov, Z. Arzoumanian, K.C. Gendreau, S. Guillot, A.K. Harding, W.C.G. Ho, J.M. Lattimer et al., The Astrophysical Journal 887, L24 (2019)

[6] B.P. Abbott et al. (LIGO Scientific, Virgo), Phys. Rev. Lett. 119, 161101 (2017), 1710.05832

[7] B. Abbott, R. Abbott, T. Abbott, F. Acernese, K. Ackley, C. Adams, T. Adams, P. Addesso, R. Adhikari, V. Adya et al., Physical Review Letters 121 (2018)

[8] L. Tolos, L. Fabbietti, Prog. Part. Nucl. Phys. 112, 103770 (2020), 2002.09223

[9] J. Haidenbauer, U.G. Meißner, A. Nogga, Eur. Phys. J. A 56, 91 (2020), 1906. 11681

[10] M.A. Lisa, S. Pratt, R. Soltz, U. Wiedemann, Ann. Rev. Nucl. Part. Sci. 55, 357 (2005)

[11] L. Fabbietti, V.M. Sarti, O.V. Doce (2020), 2012.09806

[12] D. Mihaylov, V. Mantovani Sarti, O. Arnold, L. Fabbietti, B. Hohlweger, A. Mathis, Eur. Phys. J. C 78, 394 (2018), 1802.08481

[13] S. Acharya et al. (ALICE), Nature 588, 232 (2020)

[14] S. Acharya et al. (ALICE), Phys. Lett. B 811, 135849 (2020), 2004.08018

[15] J. Haidenbauer, S. Petschauer, N. Kaiser, U.G. Meißner, A. Nogga, W. Weise, Nucl. Phys. A 915, 24 (2013), 1304.5339

[16] H. Polinder, J. Haidenbauer, U.G. Meißner, Nucl. Phys. A 779, 244 (2006), nucl-th/0605050

[17] S. Acharya et al. (ALICE) (2021), 2104.04427

[18] S. Acharya et al. (ALICE), Phys. Lett. B 805, 135419 (2020), 1910. 14407

[19] K. Sasaki et al. (HAL QCD), Nucl. Phys. A 998, 121737 (2020), 1912.08630

[20] S. Acharya et al. (ALICE), Phys. Rev. Lett. 123, 112002 (2019), 1904.12198

[21] T. Inoue (LATTICE-HALQCD), PoS INPC2016, 277 (2016), 1612.08399

[22] S. Weissenborn, D. Chatterjee, J. Schaffner-Bielich, Phys. Rev. C 85, 065802 (2012), [Erratum: Phys.Rev.C 90, 019904 (2014)], 1112.0234

[23] N. Hornick, L. Tolos, A. Zacchi, J.E. Christian, J. Schaffner-Bielich, Phys. Rev. C 98, 065804 (2018), 1808.06808

[24] S. Acharya et al. (ALICE Collaboration), ALICE-PUBLIC-2020-005, CERN-LHCC2020-018 (2020)

[25] R. Del Grande, L. Šerkšnytè, L. Fabbietti, V.M. Sarti, D. Mihaylov (2021), 2107 . 10227 